Material and methods The interventional program was implemented at the Pediatric Division of Assaf Harofeh Medical Center and included presentations to the healthcare professionals, posters, creating new ADRs reporting methods and alerts.

Results Reporting rate of ADRs during the year before implementation of the program was negligible. During the study period (three months), the reporting rate increased dramatically. A new signal was reported; two neonates with apnea related to IVIG administration in the neonatology department. The distribution of reports was: $50 \%$ from the general pediatric department, 26\% - pediatric neurology department, 21\% and $6 \%$, from the pediatric and neonatal intensive care units, respectively. Steroids and antibiotics were responsible for various ADRs, but not for a new signal.

Conclusion There is one case report in the literature on apnea related to IVIG administration. Adverse reactions in children are a major problem and sometimes can lead to morbidity mortality. Identifying new signal in pediatric patients is not common, and very challenging.

Disclosure(s) Nothing to disclose

\section{P15 LACOSAMIDE MONITORING IN THE SERUM OF CHILDREN WITH REFRACTORY EPILEPSY}

${ }^{1} \mathrm{~N}$ Kronenfeld, ${ }^{1} \mathrm{E}$ Kohn, ${ }^{2} \mathrm{M}$ Lezinger, ${ }^{3} \mathrm{~N}$ Brandriss, ${ }^{2} \mathrm{E}$ Heyman, ${ }^{3} \mathrm{~A}$ Bar-Chaim, 1,4 M Berkovitch*. 'Clinical Pharmacology and Toxicology; ${ }^{2}$ Pediatric Neurology Department; ${ }^{3}$ Laboratories Department, Biochemistry lab, Assaf Harofeh Medical Centre, Zerifin; ${ }^{4}$ Sackler Faculty of Medicine, Tel-Aviv University, Tel Aviv, Israel

\subsection{6/archdischild-2019-esdppp.54}

Background Lacosamide is indicated for various types of refractory epilepsy and as adjunctive therapy to other antiepileptic medications. Data on monitoring serum levels of lacosamide in pediatric patients is scarce.

Objective To evaluate the correlation between serum levels of lacosamide and the tolerability in children with refractory epilepsy.

Methods The medical records of 22 children with refractory epilepsy treated with lacosamide at Assaf Harofeh Medical Center were reviewed. Trough serum levels of lacosamide was measured using HPLC and correlated with its efficacy and safety.

Results Mean age of the children was $11 \pm 4$ (3-18) years. Median lacosamide daily dose was $9.3(6.6-11) \mathrm{mg} / \mathrm{kg}$ and median plasma concentration was 7.1 (5.9-11.9) ug/ml. The therapeutic range of lacosamide serum concentration is 10 to $20 \mathrm{ug} / \mathrm{ml}$. No change in seizures frequency was reported in $21.4 \%$ of children with lacosamide concentrations below $10 \mathrm{ug} / \mathrm{ml}$. However, in $40 \%$ of the children, reduction of the seizures frequency was reported when serum concentration was above $10 \mathrm{ug} / \mathrm{ml}$. No serious adverse events were reported during therapy. The prospective part of the study was initiated, and the first patients were recruited.
Conclusion Large studies, preferably prospective, on lacosamide serum monitoring including information on correlation with efficacy and safety are warranted.

Disclosure(s) Nothing to disclose

\section{P16 TOWARDS DETERMINATION OF ENDOGENOUS PRORENIN IN PAEDIATRIC SAMPLES USING A HYBRID APPROACH - IMPACT OF ANTIBODY SELECTION FOR IMMUNOCAPTURE}

I Burdman, BB Burckhardt. Institute of Clinical Pharmacy and Pharmacotherapy, Heinrich Heine University, Dusseldorf, Germany

\subsection{6/archdischild-2019-esdppp.55}

Background Since sample volume is limited in children, innovative bioanalytical methods and enrichment procedures are highly required. The analysis of endogenous substances by liquid chromatography coupled to mass spectrometry is a highly specific method because of its selectivity and accuracy. However reliable detection of endogenous substances can only be achieved by a hybrid assay approach combining immunocapture and mass spectrometry. Key element of the immunocapture procedure is the selection of the appropriate antibody for capturing the desired antigen. This study is meant to identify the most suitable antibody that facilitates the development of an hybrid assay approach concerning reliable detection of endogenous prorenin in paediatric samples.

Methods Dynabeads magnetic beads were coupled to three different antibodies from three different vendors (GeneTex, Molecular Innovations, R\&D systems). $500 \mu \mathrm{L}$ human plasma which was spiked with $20 \mathrm{ng}$ recombinant human prorenin (Cayman chemicals). The immunocapture step was followed by protease digestion and a custom-made $\mu$ elution solid-phase extraction protocol. The digest was analyzed by Shimadzu Nexera LC-system coupled with Sciex TripleTOF 6600 mass spectrometer.

Results The analysis of the captured prorenin was performed by the surrogate peptide approach. In this case the surrogate peptide was identified as unique. The comparison of the three available antibodies showed that one antibody did not ensure reliable binding properties in human matrix. Among the two remaining antibodies only one showed sufficient binding capacities to be applied in small sample volumes commonly available in paediatric samples. Using this hybrid approach enabled the enrichment of the required volume by factor of 20.

Conclusion This study identified the most suitable antibody for the immunocapture procedure of the prorenin hybrid approach. This is now followed by further mass spectrometric method development and validation prior to its application in paediatric samples.

Disclosure(s) Declaration of interest: none Ilja Burdman and Bjoern B. Burckhardt declare that there is no conflict of interest. This research did not receive any specific grant from funding agencies in the public, commercial, or not-for-profit sectors 